\title{
EVOLUCIÓN DE LOS CONCEPTOS \\ ÁCIDO-BASE A LO LARGO DE LA \\ ENSENANZA MEDIA
}

BARDANCA, M. ${ }^{\mathrm{t}-2}$, NIETO, M..$^{2-3}$ Y RODRÍGUEZ, M.C..$^{3}$

I Universidad de la República. Facultad de Química. General Flores 2124. CC 1157. Montevideo.

República Oriental del Uruguay.

2 Centro de Formación Docente. Instituto de Profesores Artigas. Av. Libertador Brig. Gral.

Lavalleja 2025. CP 11800. Montevideo. República Oriental del Uruguay.

3 Enseñanza Secundaria. Liceo núm. 2 «Hector Miranda» C. Bacigalupi 2244. CP 11800.

Montevideo. República Oriental del Uruguay.

\section{SUMMARY}

The aim of this paper is to know the evolution of the concept of acids and bases throughout the different courses of Secondary School.

\section{INTRODUCCIÓN}

Muchos son Ios temas que están incluidos en los programas de química de enseñanza media que son y serán el fundamento teórico y explicación de hechos de nuestro diario vivir.

De dichos tópicos se ha elegido el tema Ácidos y Bases con el fin de conocer los preconceptos y la evolución de los conocimientos de los estudiantes a lo largo de todos los cursos de química de enseñanza secundaria en la República Oriental del Uruguay. Ésta consta de un ciclo básico único y obligatorio (primero, segundo y tercer año) y un bachillerato diversificado (cuarto, quinto y sexto); en esta última etapa existen tres orientaciones: biológica, científica y humanística.

Los cursos de química comienzan en tercer año; existen en primero y segundo cursos de introducción a las ciencias físicas.

El tema ácidos y bases es estudiado por primera vez al final del curso de tercer año, siendo sus objetivos en este nivel:

a) identificar mediante reactivos indicadores el medio ácido o básico,

b) formular y nombrar los ácidos e hidróxidos más usados.

En cuarto año se profundiza el tema haciendo hincapié en la ionización de los mismos y en reacciones de formación de sales. Por último, en sexto año se estudian las teorías de ácidos y bases: Arrhenius, Brönsted, Lewis, teorías de indicadores, neutralización y valoración.

\section{DISEÑO DE TRABAJO}

Nos pareció importante conocer:

a) las ideas previas que tienen los estudiantes de estos importantes conceptos,

b) la eficacia de nuestros métodos de enseñanza,

c) el nivel de conocimientos en que se encuentran los estudiantes cuando concluyen la enseñanza media.

La complejidad de los objetivos de este trabajo hace que los instrumentos normalmente utilizados siempre sean insuficientes para cumplirlos completamente.

Algunos autores recurren muchas veces a las entrevistas individuales, aunque otros, como Bart (1972), Lawson (1978), Shayer (1979), han desarrollado otros tipos de pruebas que permiten obtener información con mayor facilidad. En este caso no se realizaron las entrevistas clínicas individuales, debido a que el tamaño de la muestra es relativamente grande y no es fácil la instrumentación para llevarlas a cabo. 
Para realizar nuestro estudio se planteó un cuestionario de cuatro preguntas que fueron propuestas a alumnos de diferentes cursos: de primero (edad promedio en liceos diurnos 12 a 13 años) a sexto año (17-18 años).

Dichas preguntas fueron las siguientes:

1) ¿Qué entiende por ácido?

2) ¿Qué entiende por sustancia básica o alcalina?

3) ¿Qué sustancias básica o alcalinas conoce?

4) ¿Son importantes los ácidos y las bases en la vida del hombre? Fundamente su respuesta.

Las preguntas fueron abiertas, muy generales y se respondieron en condiciones normales de clase, sin previo aviso y en forma anónima; con ello se buscó que los estudiantes contestaran libre y espontáneamente sin sugerir ni limitar respuestas y así tener un amplio espectro de ideas que nos puedan conducir a conclusiones ciertas y valederas (Paris 1989).

Se pedía a los alumnos que indicaran su edad y el sexo con el fin de establecer si había alguna tendencia con alguno de estos factores.

También nos interesaba saber si el nivel social de los estudiantes incidía en las respuestas; para ello se eligieron grupos de liceos oficiales, tanto diurnos como nocturnos, y liceos privados de distintas zonas de la ciudad de Montevideo.

En cada uno de los niveles se encuestaron alrededor de 120 alumnos pertenecientes a varios grupos.

Los cuestionarios fueron propuestos en los años 1990 y 1991 con el fin de tener resultados representativos y eliminar cualquier factor fortuito de una generación.

En todos los casos el cuestionario se propuso antes de desarrollar el tema en el curso correspondiente.

\section{RESULTADOS OBTENIDOS}

1) Concepto de ácido.

\begin{tabular}{|crrrrc|}
\hline Curso & $\begin{array}{c}\text { Respuestas } \\
\text { correctas }\end{array}$ & $\begin{array}{c}\text { Sust. peli- } \\
\text { grosa o } \\
\text { que quema } \\
(\%)\end{array}$ & $\begin{array}{c}\text { Otras } \\
\text { respuestas }\end{array}$ & $\begin{array}{c}\text { No } \\
\text { contestan }\end{array}$ & $\begin{array}{c}\text { Núm. de } \\
\text { alumnos }\end{array}$ \\
\hline $1^{2}$ & 0 & 80,0 & 7,7 & 12,3 & 115 \\
$2^{2}$ & 11,3 & 37,0 & 40,7 & 11,0 & 112 \\
$3^{9}$ & 1,5 & 67,8 & 23,0 & 7,7 & 119 \\
$4^{2}$ & 32,9 & 22,9 & 27,1 & 17,1 & 121 \\
$5^{9}$ & 40,0 & 18,0 & 20,0 & 22,0 & 126 \\
$6^{9}$ & 36,0 & 8,0 & 44,0 & 12,0 & 120 \\
\hline Magist. & 0 & 43,7 & 34,8 & 21,5 & 23 \\
\hline Facul. & 100 & 0 & 0 & 0 & 80 \\
Quím. & & & & & \\
\hline
\end{tabular}

2) Concepto de base.

\begin{tabular}{|c|c|c|c|c|}
\hline Curso & $\begin{array}{c}\text { Respuestas } \\
\text { correctas } \\
(\%)\end{array}$ & $\begin{array}{c}\text { Otras acepciones } \\
\text { de básico } \\
\text { (esencialidad) } \\
(\%)\end{array}$ & $\begin{array}{c}\text { Otras } \\
\text { respuestas } \\
(\%)\end{array}$ & $\begin{array}{c}\text { No } \\
\text { contestan } \\
(\%)\end{array}$ \\
\hline $\begin{array}{l}I^{8} \\
2^{9} \\
3^{\circ} \\
4^{\circ} \\
5^{\circ} \\
6^{9}\end{array}$ & $\begin{array}{r}0 \\
7,4 \\
4,2 \\
25,7 \\
32,0 \\
50,0\end{array}$ & $\begin{array}{r}58,5 \\
33,3 \\
23,0 \\
11,4 \\
16,0 \\
4,0\end{array}$ & $\begin{array}{r}18,5 \\
26,0 \\
32,8 \\
27,1 \\
8,0 \\
28,0\end{array}$ & $\begin{array}{l}23,0 \\
33,3 \\
40,0 \\
35,8 \\
44,0 \\
18,0\end{array}$ \\
\hline Magist. & 0 & 21,7 & 17,4 & 60,9 \\
\hline $\begin{array}{l}\text { Facuitt. } \\
\text { Quím. }\end{array}$ & 100 & 0 & 0 & 0 \\
\hline
\end{tabular}

3) Ejemplos de sustancias básicas o alcalinas.

\begin{tabular}{|lcccccccc|}
\hline Curso & $1^{8}$ & $2^{2}$ & $3^{8}$ & $4^{8}$ & $5^{\circ}$ & $6^{9}$ & Mag. & $\begin{array}{l}\text { Facul. } \\
\text { Qufm. }\end{array}$ \\
\hline $\begin{array}{l}\text { \% respuestas } \\
\text { Correctas }\end{array}$ & 0 & 7,4 & 5,5 & 14,0 & 25,4 & 77,8 & 0 & 100 \\
\hline
\end{tabular}

4) Importancia de los ácidos y las bases en la vida del hombre.

\begin{tabular}{|c|c|c|c|c|c|c|}
\hline Curso & $\begin{array}{l}\text { Imp. } \\
\text { biolo- } \\
\text { gica } \\
(\%)\end{array}$ & $\begin{array}{l}\text { Imp. } \\
\text { Industrial } \\
\text { (\%) }\end{array}$ & $\begin{array}{c}\text { Uso } \\
\text { en Ia } \\
\text { vida } \\
\text { cotidiana } \\
(\%)\end{array}$ & $\begin{array}{l}\text { Perjudi- } \\
\text { ciales } \\
\text { (\%) }\end{array}$ & $\begin{array}{c}\text { Otras } \\
\text { respues- } \\
\text { tas } \\
(\%)\end{array}$ & $\begin{array}{l}\text { No } \\
\text { contes- } \\
\text { tan } \\
(\%)\end{array}$ \\
\hline $\begin{array}{l}1^{9} \\
2^{9} \\
3^{9} \\
4^{9} \\
5^{9} \\
6^{9}\end{array}$ & $\begin{array}{l}33,8 \\
29,6 \\
30,8 \\
44,3 \\
26,0 \\
50,0\end{array}$ & $\begin{array}{c}21,5 \\
2,6 \\
4,6 \\
4,3 \\
12,7 \\
18,0\end{array}$ & $\begin{array}{l}0,0 \\
5,5 \\
0,0 \\
5,7 \\
6,0 \\
4,0\end{array}$ & $\begin{array}{c}12,3 \\
3,7 \\
0,0 \\
1,4 \\
4,0 \\
12,0\end{array}$ & $\begin{array}{c}32,4 \\
29,0 \\
27,7 \\
5,7 \\
5,3 \\
0,0\end{array}$ & $\begin{array}{c}0,0 \\
29,6 \\
36,9 \\
38,6 \\
46,0 \\
16,0\end{array}$ \\
\hline Mag. & 13,1 & 4,3 & 4,3 & 17,4 & 0,0 & 60,9 \\
\hline $\mathrm{FQ}$ & 63,6 & 18,3 & 13,6 & 0,0 & 0,0 & 4,5 \\
\hline
\end{tabular}

En su mayor parte, los estudiantes responden a un mismo patrón de pensamiento, entre diferentes grupos del mismo nivel las respuestas son concordantes independientemente del sexo, la edad y la capa social a la que pertenecen (Solomon 1987).

Se consideran como respuestas correctas aquéllas que safisfacen los objetivos del nivel inmediatamente anterior al cursado en el momento en que se propuso el cuestionario, por ejemplo en cuarto año se aceptaron como respuestas correctas:

- sustancias cuya solución acuosa posea pH inferior a 7 , - sustancias que viran al rojo el tornasol,

- sustancias que provienen de la reacción entre algunos óxidos no metálicos y el agua. 
A continuación se destacan algunos ejemplos que fueron considerados en el análisis de los resultados como otras respuestas:

\begin{tabular}{|c|}
\hline $\begin{array}{l}\text { Para ácidos } \\
\text { - Algo que me hace recordar el pornelo o el limón ( } 2^{2} \text { año). } \\
\text { - Sustancia que ataca el mámol (3r año). } \\
\text { - Sustancia que conserva alimentos (3r año). } \\
\text { - Sustancia que corroe los metales ( } 4^{q} \text { año). } \\
\text { - Sustancia química orgánica o inorgánica ( } 5^{e} \text { año). } \\
\text { - Sustancia que actúa como disolvente }\left(6^{a} \text { año). }\right.\end{array}$ \\
\hline $\begin{array}{l}\text { Para bases o alcalis } \\
\text { - Sustancia básica neutralizada por ácidos ( } 2^{2} \text { año). } \\
\text { - Imagino que es una sustancia de la cual parten otras sustan- } \\
\text { cias y que es base de éstas ( } 2^{2} \text { año). } \\
\text { - Sustancias que concentrân energía ( } 3 \mathrm{r} \text { año). } \\
\text { - Todo lo contrario a un ácido ( } 3 \mathrm{r} \text { año). } \\
\text { - Es una sustancia que no es ácida ( } 4^{2} \text { año). } \\
\text { - Sustancias que están presentes en las pilas ( } 6^{9} \text { año). }\end{array}$ \\
\hline
\end{tabular}

\section{INTERPRETACIÓN DE LOS RESULTADOS}

Hay en la actualidad un gran interés por conocer cuáles son las ideas previas de los alumnos, ya que según las modernas teorías sobre el aprendizaje sólo se logra que éste sea significativo si se parte del conocimiento de estos preconceptos para construir nuevos esquemas conceptuales (Driver 1986, Driver 1985, Hierrezuelo 1988, Anderson 1986).

El alumno estudia por primera vez ácidos y bases en la última parte del curso de química en tercer año de enseñanza secundaria, por lo tanto, los resultados obtenidos en primero, segundo y tercer año son indicativos de los preconceptos que poseen los estudiantes.

De los resultados correspondientes a las preguntas 1 y 2 , podemos decir que en esta etapa el estudiante no da respuestas corrrectas lo cual es razonable, a excepción del segundo año en que se obtienen resultados que a primera vista parecen no ser coherentes, pues; hay un $11,3 \%$ de respuestas correctas a pesar de que estos alumnos nunca estudiaron el tema.

Eso nos llev6 a pensar que a los estudiantes se les suministra cierta información en otras asignaturas que están cursando simultáneamente con química como, por ejemplo, biología u otra asignatura afín; pero esa información no es asimilada porque en tercer año ya no está presente.

De las respuestas correctas correspondientes a la primera pregunta se observa lo siguiente:

- En Ios primeros cursos predominan las definiciones operacionales o experimentales (p.e. reacción frente a metales y otras sustancias, identificación mediante reactivos indicadores de $\mathrm{pH}$ ).

- En cuarto año predominan definiciones basadas en estructura química de los ácidos (p.e. compuestos que poseen hidrógeno, un no metal y, con frecuencia, oxígeno), otros recurren también a la escala de $\mathrm{pH}$. Se supone que esto se debe a que en tercer año el alumno estudia por primera vez formulación y nomenclatura de compuestos inorgánicos y escala de $\mathrm{pH}$ en forma simplificada.

- En los cursos superiores de enseñanza secundaria, la definición predominante corresponde a la de la teoría de Arhenius.

Este resultado nos llevó a realizar la encuesta en el último año universitario de la carrera de Químico Farmacéutico (quinto año) para ver cuál era la tendencia de las respuestas en los cursos terminales de enseñanza superior. Se obtuvieron los siguientes resultados: un 7\% los definen de acuerdo a la teoría de Arrhenius, un 7\% según la teoría de Lewis, un 33\% según la teoría de Brönsted Lowry y un $53 \%$ plantean definiciones de acuerdo con las dos últimas teorías. En casi todos los casos, los estudiantes manifiestan el conocimiento de la existencia de varias teorías, pero se inclinan por una o dos de ellas. Estos resultados coinciden con los obtenidos por Cros y otros (1986, 1988).

Por otra parte son de destacar los altos porcentajes de respuestas en las que se puntualiza que ácidos son sustancias peligrosas que queman (un $80 \%$ en $1 \mathrm{r}$ año). Muy probablemente esta idea fue adquirida cuando realizaron algún experimento escolar, prevaleciendo como concepto las posibles recomendaciones que el docente hace en la clase para impedir accidentes. Esto nos llevó a plantear el cuestionario a un grupo de estudiantes del Centro de Formación Docente (Magisterio), obteniéndose prácticamente las mismas respuestas de los estudiantes encuestados de primer año.

Es importante destacar que los estudiantes no consideran las bases como sustancias peligrosas.

A partir de cuarto año, el porcentaje de este tipo de respuestas disminuye marcadamente y es en realidad muy bajo en el último año de la enseñanza media.

Conviene aclarar que en nuestro país el Centro de Formación Docente, no dependiente de la universidad, tiene dos orientaciones:

1) Instituto Magisterial, que otorga al finalizar los estudios el título habilitante para ejercer docencia a nivel primario (niños entre 3-12 años).

2) Instituto de Profesores Artigas, el cual otorga el título para ejercer la docencia especializada en cada una de las asignaturas que se dictan a nivel de enseñanza secundaria (12-18 años).

De acuerdo con las respuestas dadas por los estudiantes en la segunda pregunta, parece que a ellos les resulta más sencillo explicar qué entienden por ácidos que explicar qué entienden por bases. 
Muchos asocian la palabra básico con esencial o fundamental. Son numerosas las palabras que poseen diferentes acepciones $y$, por lo tanto, pueden tener diferentes significados, según quien las lea, escuche o diga; por lo tanto, cuando se enseña-aprende una ciencia, hay que ser muy cuidadoso en el empleo adecuado del lenguaje (Jiménez 1987, Llorens 1989).

Los porcentajes correctos, en general, fueron muy bajos, sólo en sexto año se alcanzó el $50 \%$ de respuestas correctas y nunca se superó este valor. En todos los cursos se puede apreciar que es muy importante el porcentaje de estudiantes que no contestan, también se observó que a medida que los estudiantes crecen en edad son más hábiles en dar respuestas vagas e imprecisas y no comprometedoras.

Debido a la confusión de conceptos, muchos estudiantes citan ejemplos de sustancias que, a su entender, son esenciales para la vida, como el agua, el aire, el oxígeno. En las respuestas obtenidas influyen las noticias del momento; por ejemplo, los estudiantes encuestados en 1991 mencionaban como sustancia esencial el petróleo, ya que la Guerra del Golfo y sus posibles consecuencias eran los titulares de la prensa. Otros, cuando ven la palabra alcalina, citan como ejemplo las pilas, por asociación de ideas, pero no tienen conocimiento del signifícado; tal es así que una misma persona cita ejemplos de básico y alcalino diferentes y totalmente incongruentes entre sí.

Entre los estudiantes que tienen claro el concepto de básico o alcalino, la mayor parte cita como único ejemplo el hidróxido de sodio. Tal vez sea por un empleo abusivo por parte de los docentes.

En sexto año resulta sorprendente que un $77,8 \%$ de estudiantes den ejemplos correctos y sin embargo no expresan claramente el concepto de sustancia básica, debiéndose probablemente a que los estudiantes realizan trabajos experimentales de carácter obligatorio y prácticas individuales en donde emplean con frecuencia algunas sustancias ácidas y básicas.

En cuanto a la importancia de los ácidos y las bases, la mayor parte de los estudiantes encuestados pondera la importancia biológica. Esto es de esperar debido a que en los cursos de biología se estudia con cierta profundidad el proceso de la digestión y además también podría influir la propaganda de antiácidos y de los beneficios e inconvenientes de ciertos analgésicos de carácter ácido en medios masivos de comunicación. Además un alto porcentaje de estudiantes menciona la importancia industrial, principalmente aquellos alumnos pertenecientes a primero, quinto y sexto curso.

Es de destacar que en primer año el $0 \%$ de estudiantes conoce lo que es un ácido, mientras que el $100 \%$ expresa claramente su importancia.

\section{CONCLUSIONES}

De acuerdo con los resultados obtenidos, comprobamos que:

- Un porcentaje considerable de Ios estudiantes no ha asimilado los conceptos ácido-base.

- Prácticamente ningún alumno menciona la importancia de las bases.

- En todos los niveles se observa una notoria influencia de la propaganda en los medios de comunicacion, hasta tal punto que muchos estudiantes al dar un concepto recurren a lo aportado por ella.

- Si bien en nuestros programas de enseñanza se tratan varias teorías de ácidos y bases, se comprueba que los alumnos recuerdan mayoritariamente la primera teoría que se les enseña (en general el docente comienza a citar las teorías en orden cronológico en el tiempo).

Es importante marcar el hecho de que con los alumnos de Magisterio, que en su casi totalidad han realizado bachillerato de orientación humanística (sin cursos de química) se obtienen resultados semejantes a los que corresponden a los primeros cursos de secundaria. Debemos pensar que el aprendizaje de ácidos y bases que realizaron a nivel de ciclo básico no fue significativo y fue rápidamente olvidado.

Esto nos indica que es necesaria una formación en ciencias a nivel de Magisterio, que permita a los docentes planificar sus actividades y transmitir a los alumnos, a nivel escolar, conceptos claros en forma rigurosa desde el punto de vista científico para no inducir a ideas erróneas en niños de corta edad, ya que es bien sabido que dichas ideas son difíciles de erradicar.

Los autores piensan que, para lograr mejores resultados, deberían tomarse en cuenta los siguientes aspectos:

- Buscar nuevas estrategias que aumenten el aprendizaje significativo teniendo presente los conceptos previos que poseen los estudiantes.

- Encontrar los mecanismos que hagan posible que el estudiante relacione en forma fluida lo que aprende en el aula con hechos de la vida cotidiana.

- Diferenciar entre el lenguaje común y el científico cuando se hace referencia a fenómenos estudiados por la ciencia.

Por otro lado creemos que puede ser beneficioso para la enseñanza de ciencias fomentar la participación de los profesores en distintos actividades que faciliten:

a) la actualización permanente en aspectos didácticos mediante el intercambio de resultados de experiencias e investigaciones,

b) la confección de nuevos materiales y estrategias. 


\section{REFERENCIAS BIBLIOGRÁFICAS}

ANDERSON, B., 1986. The experimental gestalt of causation: a common core to pupils' preconceptions in science, European Journal of Science Education, 8 (2), pp. 155-171.

BART, W.H., 1972. Construction and validation of formal reason instrument, Psychological Review, 30, pp. 663-670

CROS, D., CHASTRETTE, M., FAYOL, M., 1988. Conceptions of second year university students of some fundamenta! notions in chemistry, International Journal of Science Education, 10 (3), pp. 331-336.

CROS, D., MAURIN, M., AMOUROUX, R., CHASTRETTE, M., LEBER, J., FAYOL, M., 1986. Conceptions of firstyear university students of the constituents of matter and the notions of acids and bases, European Journal of Science Education, 8 (3), pp. 305-313.

DRIVER, R., 1986. Psicología cognoscitiva y esquemas conceptuales de los alumnos, Enseñanza de las Ciencias, 4(1), pp. 3-15.

DRIVER, R., GUESNE, E., y TIBERGHIEN, A., 1985.Children's ideas in science (Open University Press: Milton Keynes).
HIERREZUELO, J., y MONTERO, A., 1988. La ciencia de los alumnos (Laia/MEC: Barcelona).

JIMENEZ, M.P., 1986. Identificacion de esquemas alternativos de los alulmnos/as por los profesores/as. $V$ Jornadas de Estudio sobre la lnvestigación en la Escuela, Sevilla.

LAWSON, A.E., 1978. The development and validation of a classroom test of formal reasoning, Journal of Research in Science Teaching, 15(1), pp. 11-24

LLORENS, J.A., DEJAIME, M.C., y LLOPIS, R., 1989. La función del ienguaje en un enfoque constructivista del aprendizaje de las ciencias, Enseñanza de las Ciencias, 7 (2), pp. 111-119.

PARIS, M., 1989. Esquemas conceptuales en torno a los ácidos y las bases. Implicaciones de la instrucción en el proceso evolutivo de estos conceptos, Enseñanza de las Ciencias, número extra (III Congreso), tomo 2, pp. 290-293.

SHAYER, M., 1979. Science Reasoning Tasks. (NFER NELSON: Windsor).

SOLOMON, J., 1987. Social Influences on the Construction of pupil's understanding of Science, Studies in Science Education, 14. pp. 63-82. 